\title{
Type-II Matrices Attached to Conference Graphs
}

\author{
ADA CHAN \\ ssachan@caltech.edu \\ Department of Mathematics, California Institute of Technology, Pasadena, CA 91125, USA \\ RIE HOSOYA \\ hrie@nt.icu.ac.jp \\ Department of Mathematics, International Christian University, 3-10-2, Osawa, Mitaka-Shi, \\ Tokyo 181-8585, Japan
}

Received February 27, 2003; Revised August 15, 2003; Accepted October 27, 2003

Abstract. We determine the Nomura algebras of the type-II matrices belonging to the Bose-Mesner algebra of a conference graph.

Keywords: association schemes, Bose-Mesner algebras, Nomura algebras, spin models, type II matrices

\section{Type-II matrices and nomura algebras}

We say that an $n \times n$ matrix $W$ with complex entries is type II if

$$
W(j, i)\left(W^{-1}\right)(i, j)=\frac{1}{n}
$$

for $i, j=1, \ldots, n$. So a type-II matrix is invertible and has no zero entry. We use $I$ and $J$ to denote the identity matrix and the matrix of all ones respectively. For each integer $n \geq 2$ and a complex number $t$ satisfying $n t^{2}+n t+1=0$, the matrix $I+t J$ is a type-II matrix. These matrices are known as the Potts models, which are examples of spin models. The matrices of spin models and four-weight spin models are also type II, see [1] and [8].

Type-II matrices arise from combinatorial objects. For instance, any Hadamard matrix is type II. Chris Godsil and the first author have shown that the Bose-Mesner algebra of any strongly regular graphs contains a type-II matrix that is not type-II equivalent to the Potts model, see [6]. Two type-II matrices $W$ and $W^{\prime}$ are type-II equivalent if $W^{\prime}=M_{1} W M_{2}$ for some monomial matrices $M_{1}$ and $M_{2}$.

In [9], Jaeger, Matsumoto and Nomura have given the construction of a Bose-Mesner algebra from a type-II matrix. Let $W$ be an $n \times n$ type-II matrix. For $i, j=1, \ldots, n$, we define a vector $\mathbf{Y}_{i j}$ in $\mathbb{C}^{n}$ with its $k$-th entry being

$$
\mathbf{Y}_{i j}(k)=\frac{W(k, i)}{W(k, j)}
$$


The Nomura algebra of $W$, denoted by $\mathcal{N}_{W}$, is defined as the set of $n \times n$ matrices for which $\mathbf{Y}_{i j}$ is an eigenvector, for $i, j=1, \ldots, n$. It is easy to see that $\mathcal{N}_{W}=\mathcal{N}_{c W}$ for any non-zero scalar $c$.

For $M \in \mathcal{N}_{W}$, we use $\Theta_{W}(M)$ to denote the matrix whose $(i, j)$-th entry equals the eigenvalue of $M$ corresponding to the eigenvector $\mathbf{Y}_{i j}$. Note that $\Theta_{W}$ is a linear map, and we use $\mathcal{N}_{W}^{\prime}$ to denote its image of $\mathcal{N}_{W}$. It follows from the definition that $W$ is a type-II matrix if and only if $W^{T}$ is type II. The following significant result due to Jaeger, Matsumoto and Nomura, Theorem 4 of [9], links a type-II matrix to a pair of Bose-Mesner algebras.

Theorem 1.1 Let $W$ be an $n \times n$ type-II matrix. Then $\mathcal{N}_{W}^{\prime}=\mathcal{N}_{W^{T}}$. Moreover, $\mathcal{N}_{W}$ and $\mathcal{N}_{W}^{\prime}$ form a formally-dual pair of Bose-Mesner algebras with $\Theta_{W}$ being a formal duality.

A Bose-Mesner algebra $\mathcal{B}$ is a commutative algebra that contains $I$ and $J$ and is closed under the transpose and the Schur product (which is entrywise multiplication of two matrices). It is well known that $\mathcal{B}$ is a Bose-Mesner algebra if and only if it is equal to the span of the matrices of some association scheme. If $\mathcal{B}=\operatorname{span}(I, J)$ then it has dimension two and we call it the trivial Bose-Mesner algebra. If $\mathcal{B}$ has dimension $n$ then it is the Bose-Mesner of a group scheme. When $\mathcal{N}_{W}$ has dimension strictly between 2 and $n$, we may get a pair of association schemes that consist of interesting combinatorial objects.

It is known that the Nomura algebra of a Potts model of order $n \geq 5$ is the trivial BoseMesner algebra. It follows from a simple counting argument that the Nomura algebra of a Hadamard matrix is trivial if its order is congruent to $4 \bmod 8$, see Section 5.2 of [9].

Chris Godsil has proved in Section 4 of [5] that a type-II matrix $W$ has two distinct entries if and only if $W=a J+(b-a) N$ for some $a \neq b$ and $N$ is the incidence matrix of a symmetric design. He has also shown that if $b \neq-a$ and $n>3$ then the Nomura algebra of $W$ is trivial. In the same paper, he has determined that a symmetric type-II matrix with constant diagonal and quadratic minimal polynomial has the form $a I+b C$ where $C$ is a regular two-graph. Using the method in [5], it can be shown that the Nomura algebra of this type-II matrix is also trivial for $n \geq 5$.

Furthermore, H. Suzuki and the second author have proved in [7] that the Nomura algebra of a type-II matrix $W$ is imprimitive if and only if $W$ is type-II equivalent to the twisted tensor product of type-II matrices.

An interesting problem is to find type-II matrices that give formally dual pairs of BoseMesner algebras of dimension strictly between 2 and $n$. Motivated by this problem, we consider the type-II matrices in the Bose-Mesner algebra of conference graphs. We report in this paper that if $n>9$ then the Nomura algebras of these type-II matrices are trivial.

\section{Conference graphs}

A conference graph $G$ is a strongly regular graph with parameter

$(4 \mu+1,2 \mu, \mu-1, \mu)$ 
for some positive integer $\mu$. The eigenvalues of $G$ are $2 \mu$ and the roots $r$ and $s$ of the equation $x^{2}+x-\mu=0$. So we have

$$
\{r, s\}=\left\{\frac{-1+\sqrt{4 \mu+1}}{2}, \frac{-1-\sqrt{4 \mu+1}}{2}\right\} .
$$

For basic facts on strongly regular graphs, see [3]. It is easy to verify that the Bose-Mesner algebra of a conference graph is formally self-dual, i.e., the matrix of eigenvalues coincides with the matrix of dual eigenvalues, see [4]. Let $A$ be the adjacency matrix of a conference graph $G$. It follows from Eq. (32) of [8] that $W_{\epsilon}=t_{0} I+t_{1} A+t_{2}(J-I-A)$ is a type-II matrix if and only if

$$
t_{1}=\epsilon t, \quad t_{2}=t^{-1}, \quad t_{0}=-\epsilon s t-s t^{-1} \quad \text { and } \quad t_{0}^{-1}=-\epsilon s t^{-1}-s t
$$

for $\epsilon= \pm 1$. It follows from Remark (ii) on page 41 of [8] that $W_{\epsilon}=-i W_{-\epsilon}$. Since $\mathcal{N}_{W}=\mathcal{N}_{c W}$ for any non-zero scalar $c$, we may assume that $\epsilon=1$. So Eq. (2.1) becomes $t_{0}=t_{0}{ }^{-1}=-s\left(t+t^{-1}\right)$ which has solutions

$$
t_{0}= \pm 1, \quad\left\{t, t^{-1}\right\}=\left\{t_{0}\left(\frac{-s^{-1}+\sqrt{s^{-2}-4}}{2}\right), t_{0}\left(\frac{-s^{-1}-\sqrt{s^{-2}-4}}{2}\right)\right\} .
$$

Again since $\mathcal{N}_{W}=\mathcal{N}_{t_{0} W}$, we may assume that $t_{0}=1$ and

$$
W=I+t A+t^{-1}(J-I-A) .
$$

Note that $t$ and $t^{-1}$ are the solutions to the quadratic $t^{2}+s^{-1} t+1=0$, so is $\bar{t}$. Since for all $\mu \geq 1$

$$
s^{-2}-4=4\left(\frac{1}{(1 \pm \sqrt{4 \mu+1})^{2}}-1\right)<0,
$$

$t$ is not a real number and we see that $\bar{t}=t^{-1}$. If $\mathbf{Y}_{i j}$ is the eigenvector of $W$ defined in Eq. (1.1), then

$$
\overline{\mathbf{Y}_{i j}}=\mathbf{Y}_{j i}
$$

\section{Nomura algebra of $W$}

A Bose-Mesner algebra $\mathcal{B}$ is a commutative matrix algebra with identity that is closed under conjugate transpose. It is semisimple and it contains a basis of pairwise orthogonal idempotents (with respect to matrix multiplication), called the principle idempotents. In addition, each principle idempotent represents the orthogonal projection on an eigenspace of the matrices in $\mathcal{B}$. See [2] and [4] for more about Bose-Mesner algebras. Let $n$ be the size of matrices in $\mathcal{B}$. When $\mathcal{B}=\operatorname{span}(I, J)$ is the trivial Bose-Mesner algebra, then $\frac{1}{n} J$ and $I-\frac{1}{n} J$ are the principle idempotents of $\mathcal{B}$ corresponding to the eigenspaces $\mathbf{1}$, i.e., the space spanned by the vector of all ones, and $\mathbf{1}^{\perp}$, i.e., the space orthogonal to $\mathbf{1}$, respectively. 
The approach we take here is to show that the Nomura algebra of $W$ has $\mathbf{1}^{\perp}$ as an eigenspace. Hence $\mathcal{N}_{W}$ has only two principle idempotents, and is therefore equal to the span of $I$ and $J$.

Let $\langle\mathbf{u}, \mathbf{v}\rangle$ denote the Hermitian product of vectors $\mathbf{u}$ and $\mathbf{v}$ in $\mathbb{C}^{n}$, i.e., $\langle\mathbf{u}, \mathbf{v}\rangle=$ $\sum_{i=1}^{n} \mathbf{u}(i) \overline{\mathbf{v}(i)}$.

Lemma 3.1 Let $W$ be the type-II matrix defined in Eq. (2.2). If the Hermitian product of $\mathbf{Y}_{\alpha \beta}$ and $\mathbf{Y}_{x \alpha}$ is non-zero for all adjacent vertices $\alpha$ and $\beta$ and for all $x \neq \alpha, \beta$, then $\mathcal{N}_{W}$ is trivial.

Proof: Now $\left\langle\mathbf{Y}_{\alpha \beta}, \mathbf{Y}_{x \alpha}\right\rangle \neq 0$ implies that $\mathbf{Y}_{\alpha \beta}$ and $\mathbf{Y}_{x \alpha}$ belong to the same eigenspace. This eigenspace, denoted by $U$, contains $\mathbf{Y}_{x \alpha}$ for all $x \neq \alpha, \beta$.

Since each vertex in $G$ has degree $2 \mu \geq 2$, the vertex $\alpha$ has a neighbour $\beta^{\prime}$ distinct from $\beta$. Applying the hypothesis to $\alpha$ and $\beta^{\prime}$, we conclude that $\mathbf{Y}_{\alpha \beta^{\prime}}$ and $\mathbf{Y}_{y \alpha}$, for all $y \neq \alpha, \beta^{\prime}$, belong to the same eigenspace denoted by $U^{\prime}$.

Since $n=4 \mu+1 \geq 5$, there exists a vertex $z$ distinct from $\alpha, \beta$ and $\beta^{\prime}$. Now $\mathbf{Y}_{z \alpha}$ belongs to both $U$ and $U^{\prime}$. Hence $U=U^{\prime}$ and it contains

$$
\mathbf{Y}_{\beta^{\prime} \alpha}, \quad \mathbf{Y}_{\beta \alpha}, \quad \text { and } \quad \mathbf{Y}_{x \alpha} \quad \text { for all } x \neq \alpha .
$$

Now

$$
S=\left\{\mathbf{Y}_{x \alpha} \mid x \neq \alpha\right\} \subseteq U
$$

is a set of $n-1$ columns of $\Delta_{\alpha} W$, where $\Delta_{\alpha}$ is the diagonal matrix with its $(i, i)$-th entry being $W(i, \alpha)^{-1}$. It follows from the definition of type-II matrix that $W$ has no zero entry and is invertible. Hence both $\Delta_{\alpha}$ and $\Delta_{\alpha} W$ are invertible. Note that $\mathbf{Y}_{\alpha \alpha}=\mathbf{1} \notin S$ is the $\alpha$-th column of $\Delta_{\alpha} W$. We see that $S \subseteq U$ is the set of $n-1$ linearly independent vectors in $\mathbf{1}^{\perp}$. Now $\mathcal{N}_{W}$ contains $J$ which has $\mathbf{1}$ and $\mathbf{1}^{\perp}$ as its eigenspaces. So $\mathbf{1}^{\perp}$ is an eigenspace of $\mathcal{N}_{W}$ and $I-\frac{1}{n} J$ is a principle idemptent of $\mathcal{N}_{W}$. Therefore $\mathcal{N}_{W}=\operatorname{span}(I, J)$ is the trivial Bose-Mesner algebra.

Let $\alpha, \beta$ and $\gamma$ be vertices of a conference graph $G$ with parameters $(4 \mu+1,2 \mu, \mu-1, \mu)$. For $x_{\alpha} \in\{\alpha, \bar{\alpha}\}, x_{\beta} \in\{\beta, \bar{\beta}\}$ and $x_{\gamma} \in\{\gamma, \bar{\gamma}\}$, we define $\Gamma_{x_{\alpha} x_{\beta} x_{\gamma}}$ to be the set of vertices that are adjacent (not adjacent) to $x_{v}$ if $x_{v}=v\left(x_{v}=\bar{v}\right.$, respectively), for $v=\alpha, \beta, \gamma$. For instance $\Gamma_{\alpha \beta \gamma}$ is the set of common neighbours of $\alpha, \beta$ and $\gamma$ while $\Gamma_{\alpha \beta \bar{\gamma}}$ is the set of common neighbours of $\alpha$ and $\beta$ that are not adjacent to $\gamma$. Now the vertex set of $G$ is partitioned into

$$
\{\alpha, \beta, \gamma\} \cup \Gamma_{\alpha \beta \gamma} \cup \Gamma_{\bar{\alpha} \beta \gamma} \cup \Gamma_{\alpha \bar{\beta} \gamma} \cup \Gamma_{\alpha \beta \bar{\gamma}} \cup \Gamma_{\alpha \bar{\beta} \bar{\gamma}} \cup \Gamma_{\bar{\alpha} \beta \bar{\gamma}} \cup \Gamma_{\bar{\alpha} \bar{\beta} \gamma} \cup \Gamma_{\bar{\alpha} \bar{\beta} \bar{\gamma}} .
$$

By Eq. (2.3), we have

$$
\mathbf{Y}_{\alpha \beta}(x) \overline{\mathbf{Y}_{\gamma \alpha}(x)}=\mathbf{Y}_{\alpha \beta}(x) \mathbf{Y}_{\alpha \gamma}(x)=\frac{W(x, \alpha)^{2}}{W(x, \beta) W(x, \gamma)} .
$$


It is easy to verify that

$$
\mathbf{Y}_{\alpha \beta}(x) \overline{\mathbf{Y}_{\gamma \alpha}(x)}= \begin{cases}1 & \text { if } x \in \Gamma_{\alpha \beta \gamma} \cup \Gamma_{\bar{\alpha} \bar{\beta} \bar{\gamma}} \\ t^{2} & \text { if } x \in \Gamma_{\alpha \beta \bar{\gamma}} \cup \Gamma_{\alpha \bar{\beta} \gamma} \\ t^{-2} & \text { if } x \in \Gamma_{\bar{\alpha} \beta \bar{\gamma}} \cup \Gamma_{\bar{\alpha} \bar{\beta} \gamma} \\ t^{4} & \text { if } x \in \Gamma_{\alpha \bar{\beta} \bar{\gamma}} \\ t^{-4} & \text { if } x \in \Gamma_{\bar{\alpha} \beta \gamma} .\end{cases}
$$

Hence the Hermitian product

$$
\begin{aligned}
\left\langle\mathbf{Y}_{\alpha \beta}, \mathbf{Y}_{\gamma \alpha}\right\rangle= & \frac{W(\alpha, \alpha)^{2}}{W(\alpha, \beta) W(\alpha, \gamma)}+\frac{W(\beta, \alpha)^{2}}{W(\beta, \beta) W(\beta, \gamma)} \\
& +\frac{W(\gamma, \alpha)^{2}}{W(\gamma, \beta) W(\gamma, \gamma)}+\left|\Gamma_{\alpha \beta \gamma} \cup \Gamma_{\bar{\alpha} \bar{\beta} \bar{\gamma}}\right|+\left|\Gamma_{\alpha \bar{\beta} \gamma} \cup \Gamma_{\alpha \beta \bar{\gamma}}\right| t^{2} \\
& +\left|\Gamma_{\bar{\alpha} \beta \bar{\gamma}} \cup \Gamma_{\bar{\alpha} \bar{\beta} \gamma}\right| t^{-2}+\left|\Gamma_{\bar{\alpha} \beta \gamma}\right| t^{-4}+\left|\Gamma_{\alpha \bar{\beta} \bar{\gamma} \bar{\gamma}}\right| t^{4} .
\end{aligned}
$$

In the following computation, we let $\alpha$ and $\beta$ be adjacent vertices in a conference graph $G$. We now check that for $\mu>2$ and for all $\gamma \neq \alpha, \beta$, the Hermitian product $\left\langle\mathbf{Y}_{\alpha \beta}, \mathbf{Y}_{\gamma \alpha}\right\rangle$ is non-zero.

We first consider the case where $\gamma$ is adjacent to both $\alpha$ and $\beta$. We have $W(\alpha, \beta)=$ $W(\alpha, \gamma)=W(\beta, \gamma)=t$. We use $\Gamma_{v}$ to denote the set of neighbours of $v$ in $G$. Then we get

$$
\begin{aligned}
\Gamma_{\alpha} & =\Gamma_{\alpha \beta \gamma} \cup \Gamma_{\alpha \bar{\beta} \gamma} \cup \Gamma_{\alpha \beta \bar{\gamma}} \cup \Gamma_{\alpha \bar{\beta} \bar{\gamma}} \cup\{\beta, \gamma\} \\
\Gamma_{\beta} & =\Gamma_{\alpha \beta \gamma} \cup \Gamma_{\bar{\alpha} \beta \gamma} \cup \Gamma_{\alpha \beta \bar{\gamma}} \cup \Gamma_{\bar{\alpha} \beta \bar{\gamma}} \cup\{\alpha, \gamma\} \\
\Gamma_{\gamma} & =\Gamma_{\alpha \beta \gamma} \cup \Gamma_{\bar{\alpha} \beta \gamma} \cup \Gamma_{\alpha \bar{\beta} \gamma} \cup \Gamma_{\bar{\alpha} \bar{\beta} \gamma} \cup\{\alpha, \beta\} \\
\Gamma_{\alpha} \cap \Gamma_{\beta} & =\Gamma_{\alpha \beta \gamma} \cup \Gamma_{\alpha \beta \bar{\gamma}} \cup\{\gamma\} \\
\Gamma_{\alpha} \cap \Gamma_{\gamma} & =\Gamma_{\alpha \beta \gamma} \cup \Gamma_{\alpha \bar{\beta} \gamma} \cup\{\beta\} \\
\Gamma_{\beta} \cap \Gamma_{\gamma} & =\Gamma_{\alpha \beta \gamma} \cup \Gamma_{\bar{\alpha} \beta \gamma} \cup\{\alpha\} \\
V(G) & =\Gamma_{\alpha \beta \gamma} \cup \Gamma_{\bar{\alpha} \beta \gamma} \cup \Gamma_{\alpha \bar{\beta} \gamma} \cup \Gamma_{\alpha \beta \bar{\gamma}} \cup \Gamma_{\alpha \bar{\beta} \bar{\gamma}} \cup \Gamma_{\bar{\alpha} \beta \bar{\gamma}} \cup \Gamma_{\bar{\alpha} \bar{\beta} \gamma} \cup \Gamma_{\bar{\alpha} \bar{\beta} \bar{\gamma}} \cup\{\alpha, \beta, \gamma\} .
\end{aligned}
$$

Now we translate the above to the following system of equations.

$$
\begin{aligned}
2 \mu & =\left|\Gamma_{\alpha \beta \gamma}\right|+\left|\Gamma_{\alpha \bar{\beta} \gamma}\right|+\left|\Gamma_{\alpha \beta \bar{\gamma}}\right|+\left|\Gamma_{\alpha \bar{\beta} \bar{\gamma}}\right|+2 \\
2 \mu & =\left|\Gamma_{\alpha \beta \gamma}\right|+\left|\Gamma_{\bar{\alpha} \beta \gamma}\right|+\left|\Gamma_{\alpha \beta \bar{\gamma}}\right|+\left|\Gamma_{\bar{\alpha} \beta \bar{\gamma}}\right|+2 \\
2 \mu= & \left|\Gamma_{\alpha \beta \gamma}\right|+\left|\Gamma_{\bar{\alpha} \beta \gamma}\right|+\left|\Gamma_{\alpha \bar{\beta} \gamma}\right|+\left|\Gamma_{\bar{\alpha} \bar{\beta} \gamma}\right|+2 \\
\mu-1= & \left|\Gamma_{\alpha \beta \gamma}\right|+\left|\Gamma_{\alpha \beta \bar{\gamma}}\right|+1 \\
\mu-1= & \left|\Gamma_{\alpha \beta \gamma}\right|+\left|\Gamma_{\alpha \bar{\beta} \gamma}\right|+1 \\
\mu-1= & \left|\Gamma_{\alpha \beta \gamma}\right|+\left|\Gamma_{\bar{\alpha} \beta \gamma}\right|+1 \\
4 \mu+1= & \left|\Gamma_{\alpha \beta \gamma}\right|+\left|\Gamma_{\bar{\alpha} \beta \gamma}\right|+\left|\Gamma_{\alpha \bar{\beta} \gamma}\right|+\left|\Gamma_{\alpha \beta \bar{\gamma}}\right|+\left|\Gamma_{\alpha \bar{\beta} \bar{\gamma} \bar{\gamma}}\right|+\left|\Gamma_{\bar{\alpha} \beta \bar{\gamma}}\right|+\left|\Gamma_{\bar{\alpha} \bar{\beta} \gamma}\right|+\left|\Gamma_{\bar{\alpha} \bar{\beta} \bar{\gamma}}\right| \\
& +3 .
\end{aligned}
$$


Solving this system of equations, we get

$$
\begin{array}{clrl} 
& \left|\Gamma_{\alpha \beta \gamma}\right|=m, & & \left|\Gamma_{\bar{\alpha} \beta \gamma}\right|=\left|\Gamma_{\alpha \bar{\beta} \gamma}\right|=\left|\Gamma_{\alpha \beta \bar{\gamma}}\right|=\left|\Gamma_{\bar{\alpha} \bar{\beta} \bar{\gamma}}\right|=\mu-2-m, \\
\text { and } & & \left|\Gamma_{\alpha \bar{\beta} \bar{\gamma}}\right|=\left|\Gamma_{\bar{\alpha} \beta \bar{\gamma}}\right|=\left|\Gamma_{\bar{\alpha} \bar{\beta} \gamma}\right|=2+m,
\end{array}
$$

for some non-negative integer $m$. Using Eq. (3.1), we have

$$
\begin{aligned}
\left\langle\mathbf{Y}_{\alpha \beta}, \mathbf{Y}_{\gamma \alpha}\right\rangle= & t^{-2}+2 t+(\mu-2)+2(\mu-2-m) t^{2}+2(m+2) t^{-2} \\
& +(\mu-2-m) t^{-4}+(m+2) t^{4}
\end{aligned}
$$

It follows from $\bar{t}=t^{-1}$ that the real part of this product is

$$
\begin{aligned}
\frac{1}{2}\left(\left\langle\mathbf{Y}_{\alpha \beta}, \mathbf{Y}_{\gamma \alpha}\right\rangle+\overline{\left\langle\mathbf{Y}_{\alpha \beta}, \mathbf{Y}_{\gamma \alpha}\right\rangle}\right)= & \frac{1}{2}\left(\left(t^{2}+t^{-2}\right)+2\left(t+t^{-1}\right)+2(\mu-2)\right. \\
& \left.+2 \mu\left(t^{2}+t^{-2}\right)+\mu\left(t^{4}+t^{-4}\right)\right) .
\end{aligned}
$$

Note that $-s\left(t+t^{-1}\right)=1$ which leads to

$$
\begin{aligned}
& t^{2}+t^{-2}=\left(t+t^{-1}\right)^{2}-2=s^{-2}-2 \text { and } \\
& t^{4}+t^{-4}=\left(t+t^{-1}\right)^{4}-4\left(t^{2}+t^{-2}\right)-6=s^{-4}-4 s^{-2}+2 .
\end{aligned}
$$

So we have

$$
\begin{aligned}
& \frac{1}{2}\left(\left\langle\mathbf{Y}_{\alpha \beta}, \mathbf{Y}_{\gamma \alpha}\right\rangle+\overline{\left\langle\mathbf{Y}_{\alpha \beta}, \mathbf{Y}_{\gamma \alpha}\right\rangle}\right) \\
& \quad=\frac{1}{2}\left(s^{-2}-2-2 s^{-1}+2 \mu-4+2 \mu\left(s^{-2}-2\right)+\mu\left(s^{-4}-4 s^{-2}+2\right)\right) \\
& \quad=\frac{-1}{2 s^{4}}\left(\left(6 s^{2}-4 s+8 \mu+3\right)\left(s^{2}+s-\mu\right)-(4 \mu+1)(3 s-2 \mu)\right) .
\end{aligned}
$$

Since $s$ is a root of $x^{2}+x-\mu=0$, the above expression equals zero if and only if

$$
s=\frac{2 \mu}{3}
$$

Substituting the above equation into $s^{2}+s-\mu=0$ yields

$$
\frac{\mu(4 \mu-3)}{9}=0
$$

The only integral solution to this equation is $\mu=0$. So if $\mu>0$ then $\left\langle\mathbf{Y}_{\alpha \beta}, \mathbf{Y}_{\gamma \alpha}\right\rangle$ is non-zero. 
Secondly, we assume that $\gamma$ is adjacent to $\alpha$ but not to $\beta$. So we have $W(\alpha, \beta)=$ $W(\alpha, \gamma)=t$ and $W(\beta, \gamma)=t^{-1}$. Similar to the first case, we can set up a system of equations like that of Eq. (3.2). Solving it, we get

$$
\begin{aligned}
\left|\Gamma_{\alpha \beta \gamma}\right|=\left|\Gamma_{\alpha \bar{\beta} \bar{\gamma}}\right|=m, & \left|\Gamma_{\bar{\alpha} \beta \gamma}\right|=\left|\Gamma_{\alpha \bar{\beta} \gamma}\right|=\left|\Gamma_{\alpha \beta \bar{\gamma}}\right|=\left|\Gamma_{\bar{\alpha} \bar{\beta} \bar{\gamma}}\right|=\mu-1-m, \\
\text { and } & \left|\Gamma_{\bar{\alpha} \beta \bar{\gamma}}\right|=\left|\Gamma_{\bar{\alpha} \bar{\beta} \gamma}\right|=m+1,
\end{aligned}
$$

for some non-negative integer $m$. By Eq. (3.1), we get

$$
\begin{aligned}
\left\langle\mathbf{Y}_{\alpha \beta}, \mathbf{Y}_{\gamma \alpha}\right\rangle= & t^{-2}+2 t^{3}+(\mu-1)+2(\mu-1-m) t^{2}+2(m+1) t^{-2} \\
& +(\mu-1-m) t^{-4}+m t^{4}
\end{aligned}
$$

and

$$
\begin{aligned}
\left\langle\mathbf{Y}_{\alpha \beta}, \mathbf{Y}_{\gamma \alpha}\right\rangle+\overline{\left\langle\mathbf{Y}_{\alpha \beta}, \mathbf{Y}_{\gamma \alpha}\right\rangle}= & \left(t^{2}+t^{-2}\right)+2\left(t^{3}+t^{-3}\right)+2(\mu-1) \\
& +2 \mu\left(t^{2}+t^{-2}\right)+(\mu-1)\left(t^{4}+t^{-4}\right) .
\end{aligned}
$$

Since $t^{3}+t^{-3}=\left(t+t^{-1}\right)^{3}-3\left(t+t^{-1}\right)=-s^{-3}+3 s^{-1}$, we get

$$
\begin{aligned}
& \left\langle\mathbf{Y}_{\alpha \beta}, \mathbf{Y}_{\gamma \alpha}\right\rangle+\overline{\left\langle\mathbf{Y}_{\alpha \beta}, \mathbf{Y}_{\gamma \alpha}\right\rangle} \\
& =\left(s^{-2}-2\right)+2\left(3 s^{-1}-s^{-3}\right)+2(\mu-1)+2 \mu\left(s^{-2}-2\right)+(\mu-1)\left(s^{-4}-4 s^{-2}+2\right) \\
& =-s^{-4}\left(\left(6 s^{2}-12 s+8 \mu+7\right)\left(s^{2}+s-\mu\right)-(4 \mu+1)(5 s-2 \mu-1)\right) .
\end{aligned}
$$

Again $s^{2}+s-\mu=0$, the above expression equals zero if and only if

$$
s=\frac{2 \mu+1}{5} .
$$

Substituting this value into $s^{2}+s-\mu$ yields

$$
\frac{(4 \mu-3)(\mu-2)}{25}=0 .
$$

The only integral solution to this equation is 2 . So if $\mu>2$ then the Hermitian product of $\mathbf{Y}_{\alpha \beta}$ and $\mathbf{Y}_{\gamma \alpha}$ is non-zero.

Thirdly, suppose $\gamma$ is adjacent to $\beta$ but not to $\alpha$. Then $W(\alpha, \gamma)=t^{-1}$ and $W(\alpha, \beta)=$ $W(\beta, \gamma)=t$. From a system of equations similar to that of Eq. (3.2), we get

$$
\begin{array}{clrl}
\left|\Gamma_{\alpha \beta \gamma}\right|=\left|\Gamma_{\bar{\alpha} \beta \bar{\gamma}}\right|=m, & & \left|\Gamma_{\bar{\alpha} \beta \gamma}\right|=\left|\Gamma_{\alpha \bar{\beta} \gamma}\right|=\left|\Gamma_{\alpha \beta \bar{\gamma}}\right|=\left|\Gamma_{\bar{\alpha} \bar{\beta} \bar{\gamma}}\right|=\mu-1-m, \\
\text { and } & \left|\Gamma_{\alpha \bar{\beta} \bar{\gamma}}\right|=\left|\Gamma_{\bar{\alpha} \bar{\beta} \gamma}\right|=m+1,
\end{array}
$$


for some non-negative integer $m$. By Eq. (3.1),

$$
\begin{aligned}
\left\langle\mathbf{Y}_{\alpha \beta}, \mathbf{Y}_{\gamma \alpha}\right\rangle= & 1+t+t^{-3}+(\mu-1)+2(\mu-1-m) t^{2}+(2 m+1) t^{-2} \\
& +(\mu-1-m) t^{-4}+(m+1) t^{4}
\end{aligned}
$$

The imaginary part of $\left\langle\mathbf{Y}_{\alpha \beta}, \mathbf{Y}_{\gamma \alpha}\right\rangle$ is

$$
\begin{aligned}
\frac{1}{2}\left(\left\langle\mathbf{Y}_{\alpha \beta}, \mathbf{Y}_{\gamma \alpha}\right\rangle-\overline{\left\langle\mathbf{Y}_{\alpha \beta}, \mathbf{Y}_{\gamma \alpha}\right\rangle}\right)= & \frac{1}{2}\left(\left(t-t^{-1}\right)-\left(t^{3}-t^{-3}\right)+(2 \mu-4 m-3)\left(t^{2}-t^{-2}\right)\right. \\
& \left.+(2 m+2-\mu)\left(t^{4}-t^{-4}\right)\right) .
\end{aligned}
$$

Note that $t^{2}-t^{-2}=\left(t-t^{-1}\right)(-s)^{-1}$,

$$
\begin{aligned}
& t^{3}-t^{-3}=\left(t-t^{-1}\right)\left(t^{2}+1+t^{-2}\right)=\left(t-t^{-1}\right)\left(s^{-2}-1\right), \quad \text { and } \\
& t^{4}-t^{-4}=\left(t-t^{-1}\right)\left(t^{3}+t+t^{-1}+t^{-3}\right)=\left(t-t^{-1}\right)\left(2 s^{-1}-s^{-3}\right) .
\end{aligned}
$$

So the imaginary part of $\left\langle\mathbf{Y}_{\alpha \beta}, \mathbf{Y}_{\gamma \alpha}\right\rangle$ equals

$$
\begin{aligned}
& =\frac{\left(t-t^{-1}\right)}{2}\left(1-\left(s^{-2}-1\right)-(2 \mu-4 m-3) s^{-1}+(2 m+2-\mu)\left(2 s^{-1}-s^{-3}\right)\right) \\
& =\frac{\left(t-t^{-1}\right)(1+2 s)}{2 s^{3}}\left(s^{2}+3 s+4 s m-2 s \mu-2 m-2+\mu\right) .
\end{aligned}
$$

Now we have seen in Section 2 that $s^{2}+s-\mu=0$, so

$$
s=-\frac{1}{2} \pm \frac{\sqrt{4 \mu+1}}{2}
$$

and $t^{-1}=\bar{t} \neq t$. So the imaginary part of $\left\langle\mathbf{Y}_{\alpha \beta}, \mathbf{Y}_{\gamma \alpha}\right\rangle$ equals zero if and only if

$$
\begin{aligned}
m & =\frac{-s^{2}-3 s-\mu+2 s \mu+2}{2(2 s-1)} \\
& =\frac{-\left(s^{2}+s-\mu\right)+2(\mu-1)(s-1)}{2(2 s-1)} . \\
& =\frac{(\mu-1)(s-1)}{2 s-1} \\
& =\frac{(\mu-1)(-3 \pm \sqrt{4 \mu+1})}{2(-2 \pm \sqrt{4 \mu+1})} \\
& =\frac{(\mu-1)}{2}\left(1-\frac{1}{(-2 \pm \sqrt{4 \mu+1})}\right) .
\end{aligned}
$$


For $\mu>1, m$ is an integer only when $4 \mu+1$ is a perfect square. Suppose $\sqrt{4 \mu+1}$ is an integer then we can write $\sqrt{4 \mu+1}=2 b+1$ for some integer $b \geq 1$. So $\mu=b^{2}+b$ and

$$
m=\frac{\left(b^{2}+b-1\right)(b-1)}{(2 b-1)}, \quad \text { or } \quad m=\frac{\left(b^{2}+b-1\right)(b+2)}{(2 b+3)} .
$$

Observe that

$$
8 m=\left(4 b^{2}+2 b-7\right)+\frac{1}{2 b-1}, \quad \text { or } \quad 8 m=\left(4 b^{2}+6 b-5\right)-\frac{1}{2 b+3},
$$

respectively. In either case, $8 m$ is not an integer for $b>1$. But $m=\left|\Gamma_{\alpha \beta \gamma}\right|$ is an integer. So the imaginary part of $\left\langle\mathbf{Y}_{\alpha \beta}, \mathbf{Y}_{\gamma \alpha}\right\rangle$ is not zero for $\mu>2$.

Lastly, suppose $\gamma$ is not adjacent to $\alpha$ nor $\beta$. So $W(\alpha, \beta)=t$ and $W(\alpha, \gamma)=W(\beta, \gamma)=$ $t^{-1}$. Using the same argument as above, we get

$$
\begin{aligned}
& \left|\Gamma_{\alpha \beta \gamma}\right|=\left|\Gamma_{\alpha \bar{\beta} \bar{\gamma}}\right|=\left|\Gamma_{\bar{\alpha} \beta \bar{\gamma}}\right|=\left|\Gamma_{\bar{\alpha} \bar{\beta} \gamma}\right|=m, \quad\left|\Gamma_{\bar{\alpha} \beta \gamma}\right|=\left|\Gamma_{\alpha \bar{\beta} \gamma}\right|=\mu-m, \quad \text { and } \\
& \left|\Gamma_{\alpha \beta \bar{\gamma}}\right|=\left|\Gamma_{\bar{\alpha} \bar{\beta} \bar{\gamma}}\right|=\mu-1-m,
\end{aligned}
$$

for some non-negative integer $m$. By Eq. (3.1), the Hermitian product

$$
\begin{aligned}
\left\langle\mathbf{Y}_{\alpha \beta}, \mathbf{Y}_{\gamma \alpha}\right\rangle= & 1+t^{3}+t^{-1}+(\mu-1)+(2 \mu-1-2 m) t^{2}+2 m t^{-2} \\
& +(\mu-m) t^{-4}+m t^{4}
\end{aligned}
$$

The imaginary part of this product is

$$
\begin{aligned}
& \frac{1}{2}\left(\left\langle\mathbf{Y}_{\alpha \beta}, \mathbf{Y}_{\gamma \alpha}\right\rangle-\overline{\left\langle\mathbf{Y}_{\alpha \beta}, \mathbf{Y}_{\gamma \alpha}\right\rangle}\right) \\
& \quad=\frac{1}{2}\left(\left(t^{3}-t^{-3}\right)-\left(t-t^{-1}\right)+(2 \mu-1-4 m)\left(t^{2}-t^{-2}\right)+(2 m-\mu)\left(t^{4}-t^{-4}\right)\right) \\
& \quad=\frac{\left(t^{-1}-t\right)(2 s+1)}{2 s^{3}}\left(\left(s^{2}+s-\mu\right)+(2 s \mu-2 s-4 s m+2 m)\right) \\
& \quad=\frac{ \pm\left(t^{-1}-t\right) \sqrt{4 \mu+1}}{s^{3}}(s(\mu-1)-(2 s-1) m)
\end{aligned}
$$

which equals zero if and only if

$$
\begin{aligned}
m & =\frac{(\mu-1) s}{2 s-1} \\
& =\frac{(\mu-1)}{2}\left(\frac{-1 \pm \sqrt{4 \mu+1}}{-2 \pm \sqrt{4 \mu+1}}\right) \\
& =\frac{(\mu-1)}{2}\left(1+\frac{1}{(-2 \pm \sqrt{4 \mu+1})}\right) .
\end{aligned}
$$


Again when $\mu>1, m$ is an integer only if $\sqrt{4 \mu+1}$ is an integer. Now suppose $\sqrt{4 \mu+1}=$ $2 b+1$ for some integer $b \geq 1$. Then $\mu=b^{2}+b$ and

$$
m=\frac{\left(b^{2}+b-1\right) b}{2 b-1}, \quad \text { or } \quad m=\frac{\left(b^{2}+b-1\right)(b+1)}{2 b+3} .
$$

Observe that

$$
8 m=\left(4 b^{2}+6 b-1\right)-\frac{1}{2 b-1}, \quad \text { or } \quad 8 m=\left(4 b^{2}+2 b-3\right)+\frac{1}{2 b+3},
$$

respectively. In either case, $8 m$ is not an integer for $b>1$. But $m=\left|\Gamma_{\alpha \beta \gamma}\right|$ is an integer, hence the imaginary part of $\left\langle\mathbf{Y}_{\alpha \beta}, \mathbf{Y}_{\gamma \alpha}\right\rangle$ is not zero for $\mu>2$.

Now we have shown that $\left\langle\mathbf{Y}_{\alpha \beta}, \mathbf{Y}_{\gamma \alpha}\right\rangle$ is non-zero for all $\gamma \neq \alpha, \beta$. Applying Lemma 3.1, we conclude that the Nomura algebra of $W$ is trivial when $\mu>2$.

Theorem 3.2 Let $W^{\prime}$ be a type-II matrix in the Bose-Mesner algebra of the conference graph with parameters $(4 \mu+1,2 \mu, \mu-1, \mu)$. If $\mu>2$ then $\mathcal{N}_{W^{\prime}}$ is trivial.

Proof: If $W^{\prime}$ is a non-zero scalar multiple of the type-II matrix $W$ defined in Equation (2.2) then it follows from Lemma 3.1 that $\mathcal{N}_{W^{\prime}}=\mathcal{N}_{W}$ is trivial. Otherwise, $W^{\prime}$ is a non-zero scalar multiple of the Potts model and $\mathcal{N}_{W^{\prime}}$ is also trivial.

When $\mu=1$, the conference graph is the cycle on five vertices, see page 671 of [3]. The type-II matrix $W$ defined by Eq. (2.2) is type-II equivalent to the cyclic type-II matrix of size five [10]. Its Nomura algebra is isomorphic to the Bose-Mesner algebra of the cyclic group of five elements.

When $\mu=2$, the conference graph is the point graph of the generalized quadrangle of order $(2,1)$ (or the $3 \times 3$ grid), see page 671 of [3]. Solving $s^{2}+s-2=0$ we have $s=1,-2$. If $s=1$ then $W$ is type-II equivalent to the tensor product of two copies of the Potts model of size three. The Nomura algebra of $W$ is isomorphic to the Bose-Mesner algebra of $C_{3} \otimes C_{3}$. If $s=-2$ then the Nomura algebra of $W$ is trivial.

\section{References}

1. E. Bannai and E. Bannai, "Generalized generalized spin models (four-weight spin models)," Pacific J. Math. 170(1) (1995), 1-16.

2. E. Bannai and T. Ito, Algebraic Combinatorics. I. Association Schemes, Benjamin/Cummings Publisher Co., Inc., Menlo Park, Calif., 1984.

3. A.E. Brouwer, "Strongly regular graphs," in The CRC Handbook of Combinatorial Designs, C.J. Colbourn and J.H. Dinitz (Eds.), CRC Press, Boca Raton, 1996, pp. 667-683.

4. C.D. Godsil, Algebraic Combinatorics, Chapman \& Hall, New York, 1993.

5. C.D. Godsil, "Type II matrices and Jones pairs," 2001. Preprints.

6. C.D. Godsil and A. Chan, "Strongly regular graphs and type II matrices," 2001. Preprints.

7. R. Hosoya and H. Suzuki, "Type II matrices and their Bose-Mesner algebras," J. Algebraic Combin. 17 (2003), 19-37. 
8. F. Jaeger, "Strongly regular graphs and spin models for the Kauffman polynomial," Geom. Dedicata 44 (1992), 23-52.

9. F. Jaeger, M. Matsumoto, and K. Nomura, "Bose-Mesner algebra related with type II matrices and spin models," J. Algebraic Combin. 8 (1998), 39-72.

10. K. Nomura, “Type II matrices of size five,” Graphs Combin. 15 (1999), 79-92. 